\title{
Ni ferrite based microstrip phased array antenna in L-band
}

\author{
Dheeraj Kumar ${ }^{1}$ and P. K. S. Pourush ${ }^{2}$ \\ ${ }^{1}$ Babasaheb Bhimrao Ambedkar University, Lucknow, UP, India \\ ${ }^{2}$ Department of Physics, Agra College, Agra, UP, India \\ kdheeraj_7@yahoo.co.in
}

\begin{abstract}
Characteristics of $\mathrm{Ni}$ ferrite based microstrip phased array antenna in terms of the switchability, radiation patterns and gain have been investigated using transmission line model. The radiation characteristics of antenna are described with a normal magnetic bias field towards the plane of ferrite substrate considering the phase excitation difference between the patch elements $\pi / 2$ and $\pi / 4$. It is observed that by properly varying the relative phases, it is possible to steer the direction of radiation. This advantage is applicable in radar, search/track applications.
\end{abstract}

Keywords: Microstrip antenna, switchable antenna, phased array antenna, ferrite.

\section{Introduction}

In the recent years the interest in microstrip antenna technology for a range of application such as communication, radar, missile and radiometry has been growing steadily. These antennas are becoming increasingly important today for both defense and commercial applications. In the collision-avoidance industries low-profile conformal antennas are needed to provide electronically steerable radiations to detect and identify obstacles and extrusions in front of moving vehicles. Upon navigation the receiver antennas need to follow and trace the motion of global

Fig. 1. Geometry \& coordinate system of $4 x 4$ microstrip phased array antenna.

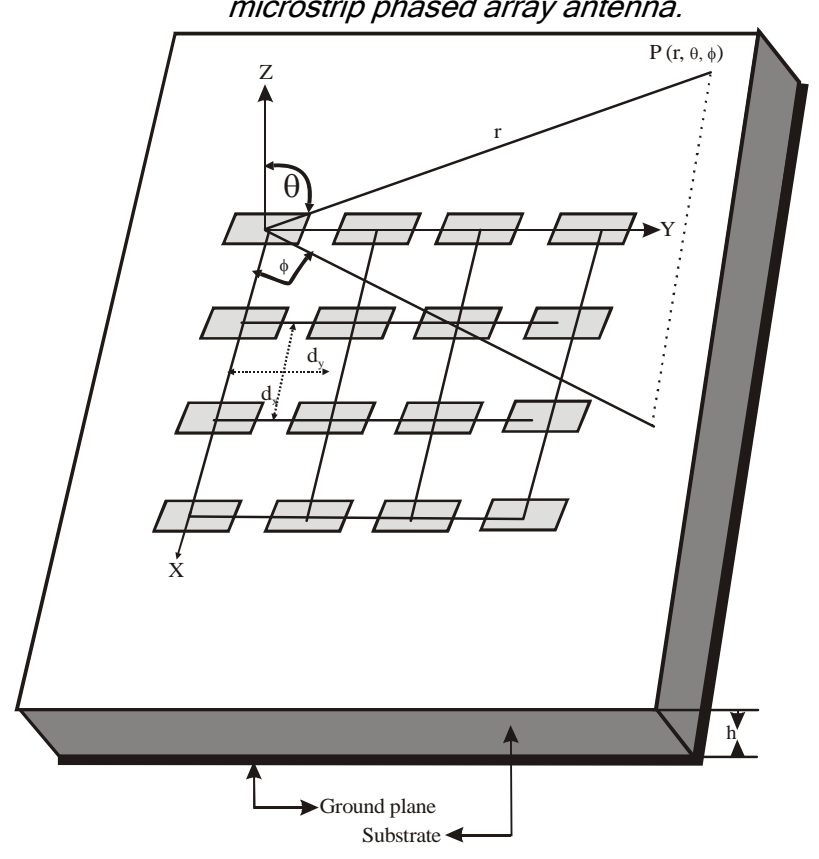

positioning system (GPS) satellites so as to continuously monitor and update their positions. Other applications can be found in target searching/tracking radars for surveillance, satellite communication systems and TV program broadcasting antennas installed with civilian jet carriers. In a phased-array system it is possible to include frequency-agile materials (ferrites) to tune and adjust the phase and amplitude of each individual element so as to compose and tailor the overall radiation into a desired pattern (Roy \& Vandon, 1992; How \& Vittoria, 2004). In the present paper, microstrip phased array antenna has been designed by taking $4 \times 4$ rectangular patch elements printed on $\mathrm{Ni}$ ferrite substrate in L-band of microwave frequency range.

\section{Theoretical analysis}

The geometry and co-ordinate system of $4 \times 4$ planar phased array of rectangular patch microstrip antenna is shown in Fig.1. It consists of 16 identical elements printed on Nickel ferrite of thickness $(\mathrm{h})=1.27 \mathrm{~mm}$ and substrate permittivity $\left(\varepsilon_{r}\right)=12$. The length and width of the patch are $7 \mathrm{~cm}$ and $2.94 \mathrm{~cm}$ respectively. Each element can be excited by a microstrip transmission line connected to the edge or by a co-axial line from the back at the plane. The bias field is taken normal to the plane of the substrate. To characterize the ferrite $z$-axis is taken along the direction of the external bias field Ho. With an $\mathrm{e}^{\mathrm{j} \omega \mathrm{t}}$ time convention, the permeability tensor of the ferrite is takes the form which is given as :

$$
[\mu]=\left[\begin{array}{ccc}
\mu & j k & 0 \\
-j k & \mu & 0 \\
0 & 0 & 1
\end{array}\right]
$$

Where $\mu$ and $\mathrm{k}$ vary with applied magnetic field and given by
Research article CIndian Society for Education and Environment (iSee)
"Ni ferrite based microstrip phased array antenna in L-band http://www.indjst.org
Dheeraj Kumar \& Pourush Indian J.Sci.Technol. 
Indian Journal of Science and Technology

$$
\begin{gathered}
\mu=1+\frac{\omega_{0}-\omega_{m}}{\omega_{0}^{2}-\omega_{m}^{2}} \\
k=\frac{\omega \omega_{m}}{\omega_{0}^{2}-\omega^{2}}
\end{gathered}
$$

Where

$\omega_{0}=\gamma H_{0}, \omega_{m}=\gamma 4 \pi M_{s} . H_{0}$ is the external bias field. $4 \pi M_{s}$ is the saturation magnetization of ferrite substrate and $\gamma$ is the gyromagnetic ratio $(\gamma=2.8 \mathrm{MHz} / \mathrm{Oe})$. When ferrite is not magnetized $\left(4 \pi M_{s}=0\right)$ it essentially acts as a dielectric (Nicholas et al., 1993). The propagation of uniform plane wave can be oriented along an arbitrary direction by changing the biasing field. The propagation may be along and perpendicular to the DC magnetic field. The dispersion relation for the two modes are obtained as Soda and Srivastava (1981).

$$
K_{e}=\left(\frac{\mu^{2}-k^{2}}{\mu}\right)^{\frac{1}{2}} K_{0}
$$

Where

$$
\begin{aligned}
& K_{0}=\frac{2 \pi f}{c} \sqrt{\varepsilon_{r}} \\
& \mu_{\text {eff }}=\frac{\mu^{2}-k^{2}}{\mu}
\end{aligned}
$$

It is noted that the mode with the propagation constant $\mathrm{K}_{0}$ is not affected by magnetic nature of the medium and propagates as it would in dielectric medium. Fig. 2 shows the dispersion curve between frequency and propagation constant of a grounded ferrite slab of thickness $h=1.27$ $\mathrm{mm}$, magnetic saturation $(4 \pi \mathrm{Ms})=2000$ Gauss and $\mathrm{H}_{0}=$ $2000 \mathrm{O}_{\mathrm{e}}$. It is evident from the figure that effective permeability of the substrate is negative for $\left[\omega_{0}\left(\omega_{0}+\right.\right.$ $\left.\left.\omega_{m}\right)\right]^{1 / 2}<\omega<\left[\omega_{0}+\omega_{m}\right]$, so there is no wave propagation in this interval, correspondingly cut off limit is at 1.2604 $\mathrm{GHz}$ and resonance is at $1.7826 \mathrm{GHz}$.

The array elements which are positioned along $x$-axis separated by a distance $d_{x}$ and those along $y$-axis are separated by a distance $d_{y}$. The total fields of the present antenna system can be expressed by the fields of a single element positioned at the origin multiplied by a factor. This is referred to as the array factor. This method is widely known as pattern multiplication approach. Since the entire array is taken as uniform, the normalized form of array factor (AF) is given as Balanis (1982).
Vol. 3 No. 12 (Dec 2010)

ISSN: 0974- 6846

$$
\begin{aligned}
& A F=0.0625 \frac{\sin \left\{2\left(K d_{x} \sin \theta \cos \phi+\beta_{x}\right)\right\}}{\sin \left\{0.5\left(K d_{x} \sin \theta \cos \phi+\beta_{x}\right)\right\}} \\
& \times \frac{\sin \left\{2\left(K d_{y} \sin \theta \sin \phi+\beta_{y}\right)\right\}}{\sin \left\{0.5\left(K d_{y} \sin \theta \sin \phi+\beta_{y}\right)\right\}}
\end{aligned}
$$

The far-zone field expressions for $4 \times 4$ planar array rectangular patch microstrip antenna are obtained as follows (Bahl \& Bhartia, 1980).

$$
\begin{aligned}
& E_{\theta}=0 \\
& E_{\phi}=-2 j V_{0} W K F(\theta, \phi) \times A F
\end{aligned}
$$

\section{Field Patterns}

The total field pattern $(\theta, \phi)$ is generally obtained from the relation.

$$
R(\theta, \phi)=\left|E_{\theta}\right|^{2}+\left|E_{\phi}\right|^{2}
$$

The values of $R(\theta, \phi)$ are computed for a case taking source frequency $\left(f_{r}\right)=2 \mathrm{GHz}$, width of the patch $(W)=$ $2.94 \mathrm{~cm}$, length of the patch $(L)=7 \mathrm{~cm}$, element separation $(\mathrm{dx}=\mathrm{dy})=1 \mathrm{~cm}$ and progressive phase excitation difference between the elements $\beta x=\beta y=\frac{\pi}{2}$ and $\frac{\pi}{4}$. The field patterns are calculated and plotted for unbiased and biased ferrite substrate in two different planes E-plane and H-plane, which are shown in Fig. 3-5.

\section{Directive gain}

The directive gain $\left(D_{g}\right)$ of the antenna of the present antenna geometry has been estimated for two values of progressive phase excitation difference $\beta x=\beta y=\frac{\pi}{2}$ and $\frac{\pi}{4} \quad$ respectively and presented in table 1. It is observed that maximum $\mathrm{Dg}=12.2 \mathrm{~dB}$ is found for this geometry under the biasing condition at $\beta_{x}$ $=\beta_{\mathrm{y}}=\pi / 2$.

\section{Discussion and conclusion}

In the present paper we have analyzed the radiation characteristics of $4 \times 4$ planar phased array of rectangular patch microstrip antenna on Nickel ferrite substrate under unbiased and biased condition at $2 \mathrm{GHz}$. The parameters used for study on biased ferrite substrate are $4 \pi \mathrm{Ms}=$ 2000 Gauss and bias field $\mathrm{H}_{0}$. $=2000 \mathrm{Oe}$ while for unbiased ferrite $4 \pi \mathrm{Ms}=0$ and $\mathrm{Ho}=0$. The progressive phase excitation difference between the element of array is considered to be $\beta_{x}=\beta_{y}=\pi / 2$ and $\pi / 4$. The radiation patterns and directive gain of the antenna system are computed and reported in Fig. 3-5 and Table1

Research article CIndian Society for Education and Environment (iSee)
"Ni ferrite based microstrip phased array antenna in L-band http://www.indjst.org
Dheeraj Kumar \& Pourush Indian J.Sci.Technol. 
Fig. 2. Variation between propagation constant \& frequency for ordinary \& extraordinary radiations.

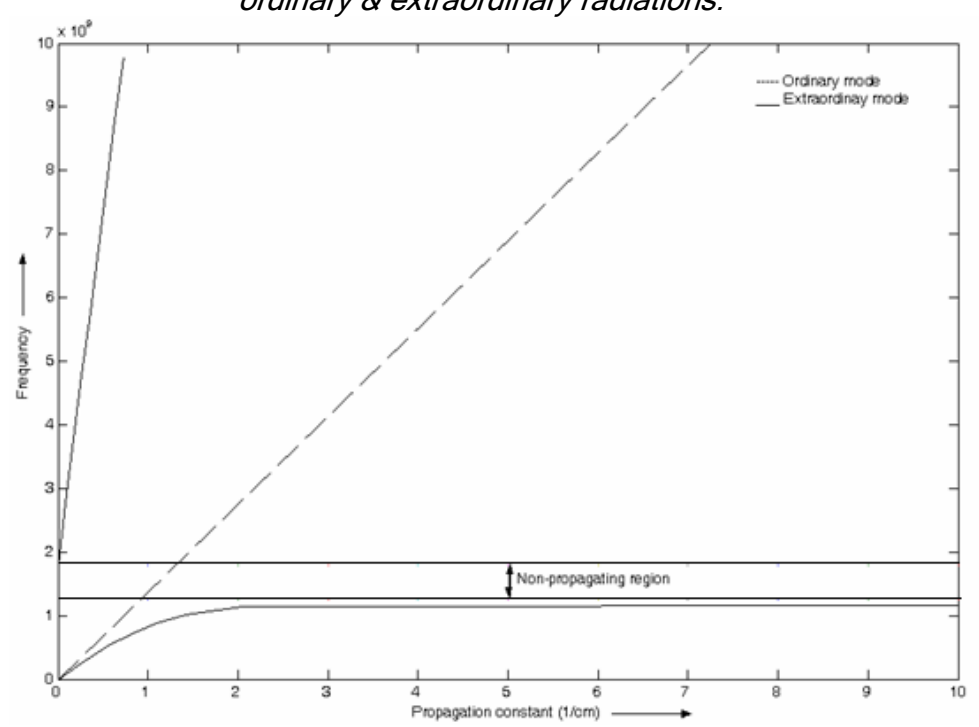

Fig. 3. Variation in field patterns of antenna in $\mathrm{H}$ plane for $\beta \mathrm{x}=\beta \mathrm{y}=\pi / 2$

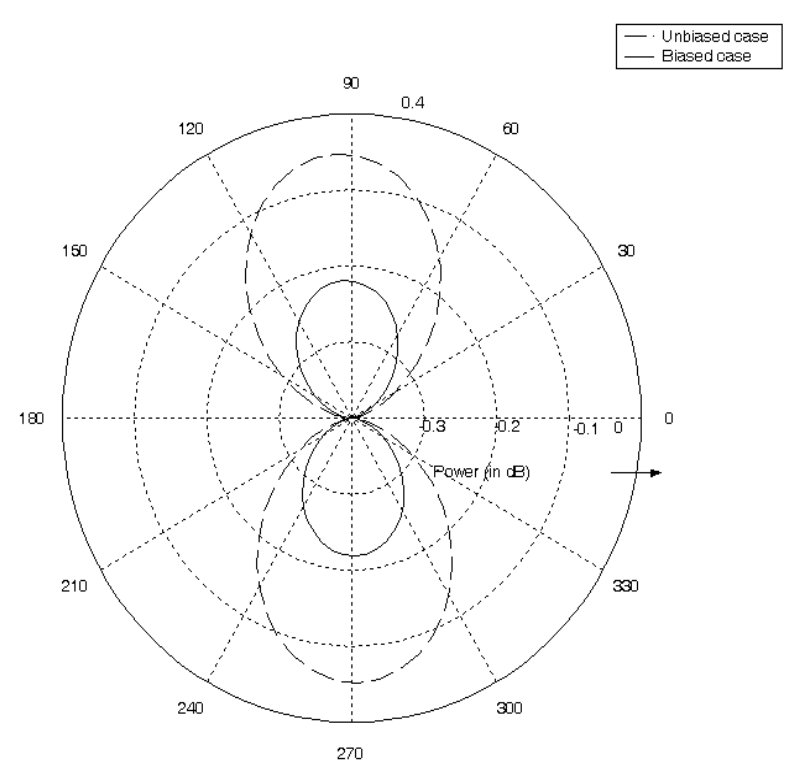

Fig. 4. Variation in field patterns of antenna in $E$ plane for $\beta x=\beta y=\pi / 4$

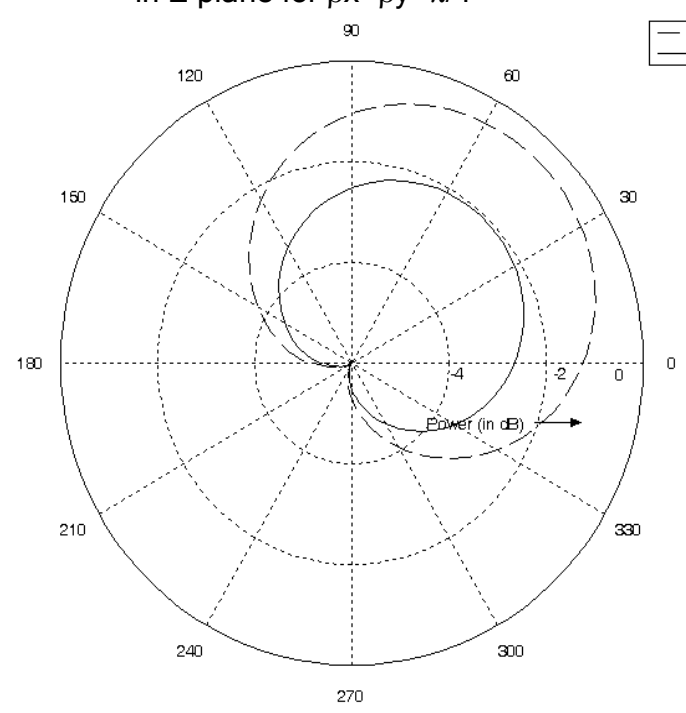

Fig. 5. Variation in field patterns of antenna in $\mathrm{H}$ plane for $\beta \mathrm{x}=\beta \mathrm{y}=\pi / 4$

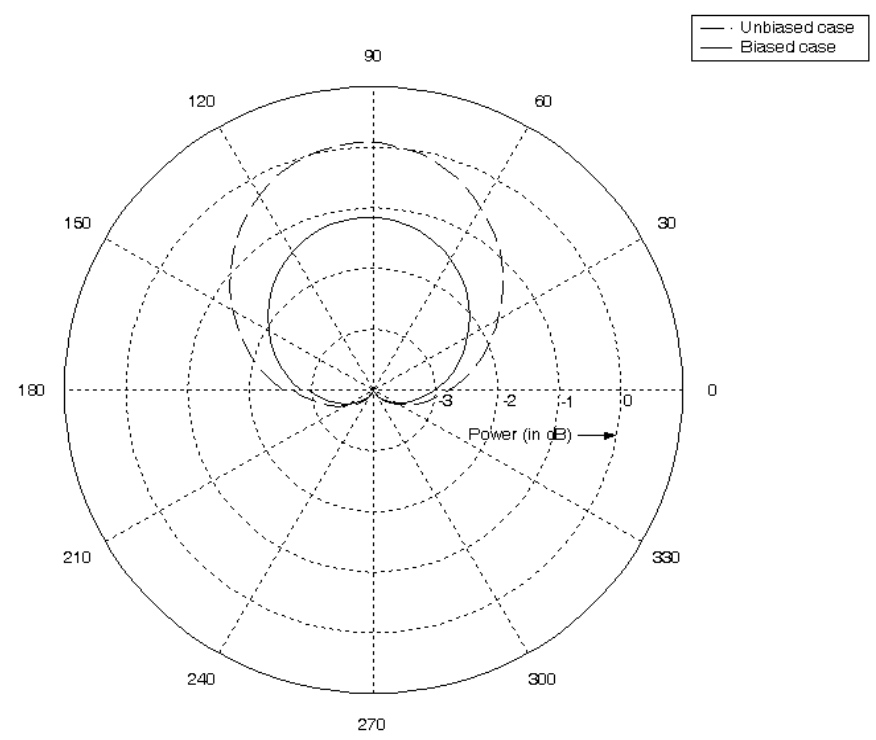

Table 1. Calculated values of directive gain.

\begin{tabular}{|c|c|c|c|c|}
\hline \multirow{2}{*}{ Antenna parameters } & \multicolumn{2}{|c|}{ Unbiased case } & \multicolumn{2}{c|}{ Biased case } \\
\cline { 2 - 5 } & $\beta \mathrm{x}=\beta \mathrm{y}=\pi / 2$ & $\beta \mathrm{x}=\beta \mathrm{y}=\pi / 4$ & $\beta \mathrm{x}=\beta \mathrm{y}=\pi / 2$ & $\beta \mathrm{x}=\beta \mathrm{y}=\pi / 4$ \\
\hline Directive gain $(\mathrm{dB})$ & 11.7 & 10.4 & 12.2 & 11.3 \\
\hline
\end{tabular}


respectively. It is found that there is a significant change in the radiation characteristics of the antenna system by the magnetization of the ferrite substrate. The direction of principal maxima of field patterns changes by changing the phase excitation difference between the elements. It is evident from the dispersion effect on ferrite material then there must be propagating and non-propagating region for antenna.

Some salient features of the antenna system are summarized as follows:

a) By the application of external magnetic field, the relative amplitude of power of the antenna changes in both the plane (i.e. E plane \& $\mathrm{H}$-plane).

b) It is observed from the dispersion curve for the given parameters the cutoff limit is $1.2604 \mathrm{GHz}$ and resonance limit is $1.7825 \mathrm{GHz}$. Within this range $\mu_{\text {eff }}$ is negative and the extra ordinary wave is decaying so antenna is effectively off as a radiator in this range. Thus by properly selecting and changing the bias field the switchable antenna can be designed and we can find out the optimum performance of the antenna system.

c) By the application of DC bias the gain of the antenna increased. In unbiased case for $\beta_{x}=\beta_{y}=\pi / 2$ the value of gain is $11.7 \mathrm{~dB}$ while for biased case gain is $12.2 \mathrm{~dB}$.

d) The relative amplitude and phase of the signals applied to each of the elements are controlled to obtain the desired radiation pattern from the combined action of all the elements.

e) By properly varying the relative phase it is possible to steer the direction of main beam. The main advantage that offered by the phased array antenna is steering the beam. This advantage is very much applicable in radar; search/track applications.
Vol. 3 No. 12 (Dec 2010)

ISSN: 0974- 6846

\section{References}

1. Bahl IJ and Bhartia P (1980) Microstrip antenna, Artech house.

2. Balanis VA (1982) Antenna theory analysis and design. John Wiley, New York.

3. How H and Vittoria C (2004) Microwave phase shifters utilizing nonreciprocal wave propagation. IEEE Trans Microwave Theory Technique. 52, 8.

4. Kumar D and Pourush PKS (2004) Triangular microstrip array on NiZn substrate in L-band. Microwave Optical Technol. Lett. 43, 4, 286290.Nicholas E, Timothy BF and Silverstein Robert S (1993) Dipole arrays printed on ferrite substrates. IEEE Trans. A \& P. 41, 165-175.

5. Roy JS and Vandon P (1992) Circularly polarized for field of an axially magnetized circular ferrite microstrip antenna. Microwave Opt. Tech. Lett. 5, 228-230.

6. Soda MS and Srivastava NC (1981) Microwave propagation in ferrimagnetics. Plenum press, New York \& London. 